\title{
Determination of Diabetic Retinopathy Using Fractals
}

\author{
Dr. V. Shanthoshini Deviha \\ Assistant Professor in Mathematics, Trichy Engineering College, Trichy, Tamilnadu
}

\begin{abstract}
The intention of this paper is to determine the retinal blood vessel system of diabetics. The retinal blood vessel system has been determined using Reynold's number and Poiseuille's law. A new algorithm has been established to traverse through the retinal blood vessel system in a robust manner.
\end{abstract}

Keywords: Fractals, Retinal blood vessel system, Reynold"s number, Poiseuille"s law

\section{Introduction}

In the recent articles fractal model for the blood vessels in different parts of human body has been analysed where as this exertion deals with fractal retinal blood vessel trees. The retinal blood vessels have been modeled using fractals. The pressure drop has been found out according to Poiseuillees law [13].

\section{Fractals}

A Fractal is an object which appears self-similar under varying degrees of magnification. Self-similarity is the major characteristic of the fractal objects [7]. Recent studies have attempted with some success to characterize certain parts of the body using fractal geometry where the retinal blood vessel system exhibits fractal characteristic. There are many definitions for fractal, among them

a) "A fractal is a shape made of parts similar to the whole in some way".

b) "A fractal is by definition for which the Hausdorff dimension strictly exceeds the Topological dimension"

Mandelbrot (1975) introduced the term "FRACTAL" to characterize spatial or temporal phenomena that are continuous but not differentiable. Fractal objects and processes are therefore said to display ,Self-invariant ${ }^{\text {ee }}$ (Selfsimilar or Self-affine) properties [5]. Fractal structures do not have a single length scale, while fractal processes (timeseries) cannot be characterized by a single-time scale [10].

\section{Retina}

The retina is a thin layer of tissue that lines the back of the eye on the inside. It is located near the optic nerve. The purpose of the retina is to receive light that the lens has focused, convert the light into neural signals, and send these signals on to the brain for visual recognition. The retina processes light through a layer of photoreceptor cells. These are essentially light-sensitive cells, responsible for detecting qualities such as color and light-intensity. The retina processes the information gathered by the photoreceptor cells and sends this information to the brain via the optic nerve.(Fig-1)

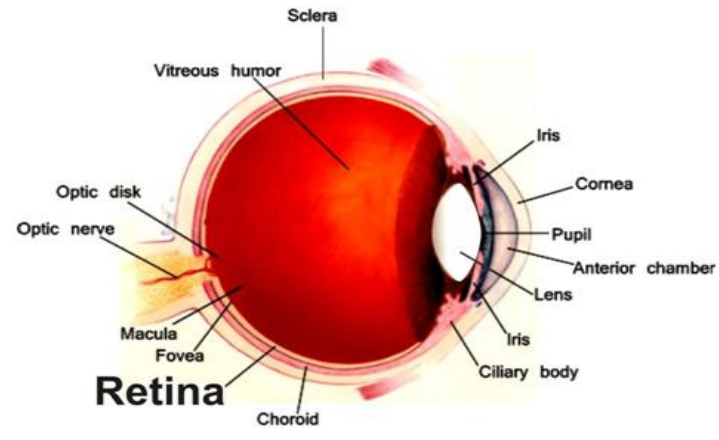

Figure 1: Structure of the eye

\section{Diabetic Retinopathy}

Diabetic retinopathy occurs as a result of high blood sugar, and can cause blindness if left untreated.Diabetic retinopathy is an eye condition that affects people with diabetes who have high blood glucose, or blood sugar, over a prolonged period of time. Too much blood sugar can damage the blood vessels in the back of the eye, preventing the retina from receiving the proper amount of nutrients it needs to maintain vision.

The retina is light-sensitive nerve tissue at the back of the eye. As light enters the front of the eye, the retina converts the light rays into electrical impulses that travel along the optic nerve to part of the brain called the visual cortex. The brain then combines images sent from both eyes to interpret them as a single, three-dimensional image. This allows us to perceive depth and distance. Without the retina, the eye cannot communicate with the brain, making vision impossible. Diabetic retinopathy occurs when diabetes damages the tiny blood vessels in the retina. In the early stages of diabetic retinopathy, called non-proliferative retinopathy, these blood vessels leak fluid and distort sight. In the more advanced stage of diabetic retinopathy, called proliferative retinopathy, fragile new blood vessels grow around the retina and in the vitreous humour. (Fig-3) 


\section{International Journal of Science and Research (IJSR) \\ ISSN (Online): 2319-7064}

Index Copernicus Value (2013): 6.14 | Impact Factor (2014): 5.611

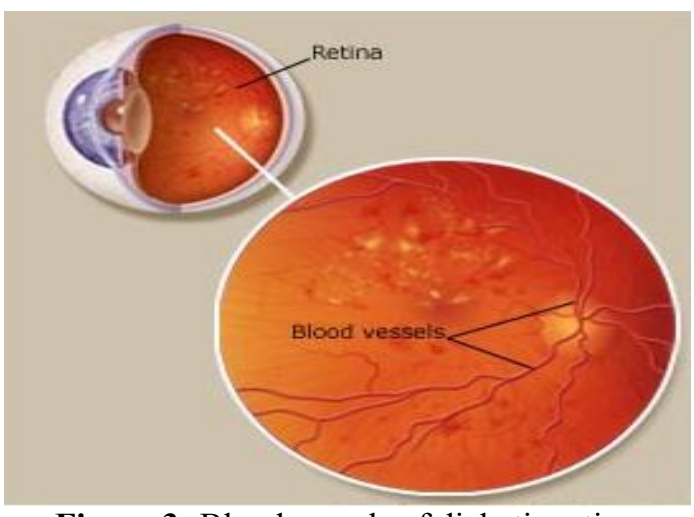

Figure 3: Blood vessels of diabetic retina

\section{Fractal model for retinal blood vessels}

Fractal model of blood vessel system is a certain geometrical simplification but it suffices for acceptable blood flow analysis [4]. This analysis permits understanding influence of hemodynamic forces.[8]. In real blood vessels system, vessel walls are elastic and change its diameters [6]. In this way resistance of blood vessel system is regulated. This process is known as autoregulation and corrects nutrition of all cells in human body [9-11]. Blood flow estimation assumes laminar flow for the entire fractal vascular tree. In large arteries a rhythmical contraction of a deviation from the normal of the laminar flow is a result of wave propogation. [2, 3, 12] (Fig.4)

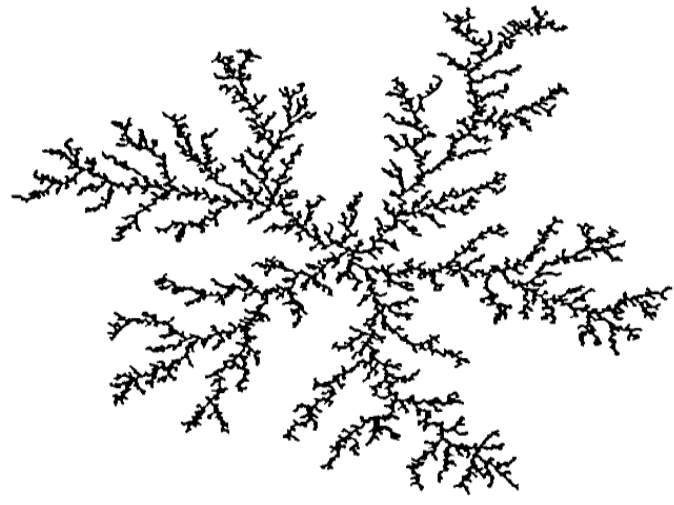

Figure 4: Fractal Vascular Tree

\section{Methods}

Blood flow in the retinal blood vessel system has been mathematically calculated by the following methods. These results are very useful to investigate the defects in the above system.

\section{Rate of flow (Q)}

It is defined as the quantity of the fluid flowing per second through a section of a pipe or a channel. For an incompressible fluid the rate of flow is expressed as the volume of fluid flowing across the section per second. Mathematically the rate of flow is defined as

$$
Q=A \times V
$$

where $Q$ is the Rate of blood flow ( $\mathrm{ml} / \mathrm{sec}), A$ is the crosssecional area $\left(\mathrm{cm}^{2}\right), V$ is the velocity of the blood.

\section{Poiseuille's law}

The quantity of blood that flows through a vessel in a given period of time i.e. per unit of time, is equal to the velocity of flow times the cross sectional area according to the following equations:

$$
Q=V \pi a^{2}
$$

$V=\left(P_{1}-P_{2}\right) a^{2} / 8 \eta L$

Now equation (1) becomes

$$
\begin{array}{r}
Q=\left\lfloor\left(P_{1}-P_{2}\right) a^{2} / 8 \eta L\right\rfloor \pi a^{2} \\
Q=\frac{\pi * a^{4} \Delta P}{8 \eta L}
\end{array}
$$

where $\Delta P$ is the pressure difference $\left(P_{1}-P_{2}\right), \eta$ is the Viscosity of the Blood (Poises), $L$ is the Length of the Vessel (Cms). The equation (2) is known as HagenPoiseuille"s equation. This equation shows that the rate of blood flow is directly proportional to the fourth power of the radius of the vessel and also illustrates that the diameter of a vessel plays a great role in determining the rate of blood flow. The most effective factor controlling blood flow is radius of the blood vessel. High blood pressure can be caused by narrowing blood vessel and is reduced by relaxing the smooth muscle tension that controls the blood vessel radius. This process is known as an auto regulation.

\section{Pressure Drop}

Pressure drop is a positive function depending on the blood flow, which passes through the different parts of the retinal blood vessel system. Pressure drop have been calculated according to Poiseuille"s law, the pressure drop $\mathrm{P}$ for rate of flow $\mathrm{Q}$ through a blood vessel with radius $a$ over a length $\mathrm{L}$ is

$$
P=\frac{8 \eta Q L}{\pi a^{4}}
$$

Pressure drop maintains the energy dissipation rate of the flow of blood for the various parts of the system (Table2).

\section{Reynold's number}

When normal laminar blood flow becomes turbulent flow

- The rate of blood flow (i.e) the velocity of flow, is high

- It passes by an obstruction in a vessel (as in case of compression by cuff of Sphygmomanometer).

- It makes a sharp turn.

- It passes over a rough surface

Turbulence is also related to the diameter of the vessel and the viscosity of the blood which can be expressed by ratio of inertia to viscous forces.

$$
R_{e}=\frac{V * L}{\eta}
$$

where $R_{\mathrm{e}}$ is the Reynold's number, $L$ is the length of the blood vessel, $V$ is the Average velocity and $\eta$ is the Viscosity of the blood. This is the most important dimensionless number; it describes the fluid flow regime. If $R_{e}$ is higher, there is a greater probability of the turbulence (Table2). 


\section{International Journal of Science and Research (IJSR) \\ ISSN (Online): 2319-7064 \\ Index Copernicus Value (2013): 6.14 | Impact Factor (2014): 5.611}

The above factors has been analysed for diabetic patient and is given in the Table 2 .

\section{Algorithm}

The algorithm traverses through the blood vessels of the retina in a recursive manner

Name: Flood Fill algorithm

Step 1 - Initialize the value of seed point (seed $\mathrm{x}$, seed $\mathrm{y}$ ), fvessel and dvessel.

Step 2 - Define the boundary values of the retina.

Step 3 - Check if the current seed point is of dvessel, then repeat the steps 4 and 5 till the

boundary is reached.

Step 4 - Change the default vessel with the fvessel at the seed point.
Step 5-Recursively follow the procedure with four neighborhood points.

FloodFill (seed $x-1$, seed y, fvessel, dvessel)

FloodFill (seed $\mathrm{x}+1$, seed $\mathrm{y}$, fvessel, dvessel)

FloodFill (seed x, seed y - 1, fvessel, dvessel)

FloodFill (seed $x-1$, seed y +1 , fvessel, dvessel)

Step 6 - Exit

The above algorithm has been programmed and run by $\mathrm{C}++$.

\section{Numerical Simulation Results}

The human retinal blood vessel system features a tremendous variety of components with different geometrical dimensions and mechanical properties. A synthetic view is given in the following Table.1.

Table 1: Data Report of diabetic patient eye

\begin{tabular}{|c|c|c|c|c|}
\hline Vessels & $\begin{array}{c}\text { Radius } \\
(\mathrm{r})(\mathrm{mm})\end{array}$ & $\begin{array}{c}\text { Total Cross Section } \\
(\mathrm{A})\left(\mathrm{mm}^{2}\right)\end{array}$ & $\begin{array}{c}\text { Average Velocity } \\
(\mathrm{V})(\mu 1 / \mathrm{min})\end{array}$ & $\begin{array}{c}\text { Length } \\
(\mathrm{L})(\mathrm{mm})\end{array}$ \\
\hline Superior Temporal Artery & $0.052 \pm 0.009$ & $0.104 \pm 0.018$ & $86 \pm 10$ & 0.2 \\
\hline Inferior Temporal Artery & $0.0550 \pm 0.0097$ & $0.103 \pm 0.015$ & $74 \pm 13$ & 0.2 \\
\hline Superior Temporal Vein & $0.0645 \pm 0.0125$ & $0.129 \pm 0.025$ & $89 \pm 12$ & 0.2 \\
\hline Inferior Temporal Vein & $0.0883 \pm 0.050$ & $0.201 \pm 0.011$ & $80 \pm 21$ & 0.2 \\
\hline Neuro Retinal Rim & $0.023 \pm 0.186$ & $2.51 \pm 0.11$ & $92 \pm 19$ & 0.2 \\
\hline
\end{tabular}

The viscosity of the blood is 5 Poise. For example, the blood vessel Superior temporal artery has been shown.

1) Area of the cross section

$$
C=\pi r^{2}
$$

$=\pi \times(0.052 \pm 0.009)^{2}$

$$
=\left(8.494867 \times 10^{-3} \pm 2.54469 \times 10^{-4}\right) \mathrm{mm}^{2}
$$

2) Rate of flow $Q=A \times V$

$$
\begin{aligned}
& =(0.104 \pm 0.018) \times(86 \pm 10) \\
& =(8.944 \pm 0.18) \mathrm{mm}^{3} / \mathrm{sec}
\end{aligned}
$$

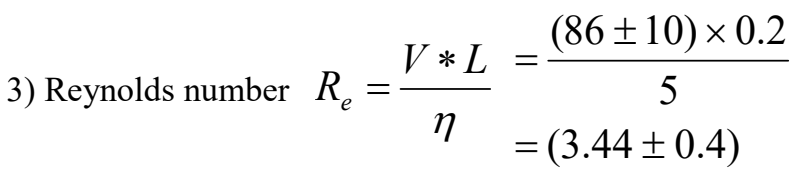

4) Pressure drop (Poiseuille"s Law) $P=\frac{8 \eta Q L}{\pi r^{4}}$

$$
\begin{aligned}
& =\frac{8 \times 5 \times(8.944 \pm 0.18) \times 0.2}{\pi \times(0.052 \pm 0.009)^{4}} \\
& =(3115003.438 \pm 69862252.11) \mathrm{mm}^{3} / \mathrm{sec}
\end{aligned}
$$

5) Total blood flow

$$
Q=\pi \Delta \mathrm{P} d^{4} / 8 \eta L
$$

$=\pi(3115003.438 \pm 69862252.11)(0.104 \pm 0.018)^{4} / 8 \times 5 \times 0.2$

$=(143.104 \pm 2.88)$ dynes $/ \mathrm{mm}^{2}$

Table 2: Analysis of Data for other parts of the retinal blood vessel system of diabetic eye

\begin{tabular}{|c|c|c|c|c|c|}
\hline Vessels & $\begin{array}{c}\text { Area of the cross section } \\
(\mathrm{C})\left(\mathrm{mm}^{2}\right)\end{array}$ & $\begin{array}{c}\text { Rate of flow }(\mathrm{Q}) \\
\left(\mathrm{mm}^{3} / \mathrm{sec}\right)\end{array}$ & $\begin{array}{c}\text { Reynolds } \\
\text { number }\left(\mathrm{R}_{\mathrm{e}}\right)\end{array}$ & $\begin{array}{c}\text { Pressure drop }(\mathrm{P}) \\
\left(\mathrm{mm}^{3} / \mathrm{sec}\right)(\text { Poiseuill } \mathrm{s} \text { Law })\end{array}$ & $\begin{array}{c}\text { Total blood flow } \\
(\mathrm{Q}) \mathrm{mm}^{3} / \mathrm{sec}\end{array}$ \\
\hline $\begin{array}{c}\text { Superior } \\
\text { Temporal Artery }\end{array}$ & $8.494867 \times 10^{-3} \pm 2.54469 \times 10^{-4}$ & $8.944 \pm 0.18$ & $3.44 \pm 0.4$ & $3115003.438 \pm 69862252.11$ & $\begin{array}{c}0.730558522 \pm \\
2.54469 \times 10^{-3}\end{array}$ \\
\hline $\begin{array}{c}\text { Inferior } \\
\text { Temporal Artery }\end{array}$ & $9.5003317 \times 10^{-3} \pm 2.95592 \times 10^{-4}$ & $7.622 \pm 0.195$ & $2.96 \pm 0.52$ & $2121086.114 \pm 56090303.33$ & $\begin{array}{c}3.224 .916059 \pm 0.11 \\
798454\end{array}$ \\
\hline $\begin{array}{c}\text { Superior } \\
\text { Temporal Vein }\end{array}$ & $1.306981 \times 10^{-2} \pm 4.908738521 \times 10^{-4}$ & $3.225 \times 10^{-3} \pm 0.3$ & $3.56 \pm 0.48$ & $4.744942727 \pm 3.1291135 .05$ & $\begin{array}{c}172.3810969 \pm \\
307200\end{array}$ \\
\hline $\begin{array}{c}\text { Inferior } \\
\text { Temporal Vein }\end{array}$ & $2.4494652 \times 10^{-2} \pm 7.853981 \times 10^{-3}$ & $16.08 \pm 2.31$ & $3.2 \pm 0.84$ & $673570.9437 \pm 941178.6715$ & $\begin{array}{c}359.496577 \pm \\
26.45106911\end{array}$ \\
\hline $\begin{array}{c}\text { Neuro Retinal } \\
\text { Rim }\end{array}$ & $1.661902 \times 10^{-3} \pm 0.108686539$ & $230.92 \pm 2.09$ & $3.68 \pm 0.76$ & $2101310928 \pm 4446.667165$ & $3694.72 \pm 33.44$ \\
\hline
\end{tabular}

\section{Conclusion}

The blood vessels have been numerically calculated for the parameters such as area of the cross section, rate of flow, Pressure drop and Reynold 's number for a diabetic patient, in our proposed system. Reynold's number provides us the accurate result of the affected blood vessels of the retinal blood vessel system in an effectual conduct. This numerical simulation helps us in understanding the affected blood vessels of the retina. The recursive strategy is a useful tool to go through the system in an effective manner. 


\section{References}

[1] Aho, Hopcroft, Ullman, The Design and Analysis of Computer Algorithms, Pearson Education Asia, 176179 (2001).

[2] Cohn.D Optimal Systems I, The Vascular System, Bull Math Biophys, 16, 59-74 (1954).

[3] Cohn.D Optimal Systems II, The Vascular System, Bull Math Biophys17, 219-227 (1955).

[4] Gilbert T. Feke, Hiroshi Tagawa, Dana M. Deupree, Douglas G. Goger, Jerry Sebag and John J. Weirer, Blood Flow In the Normal Human Retina, Investigative Ophthalmology \& Visual Science, Vol. 30, No. 1, 58-65 (1989)

[5] Hastings.H.M and G.Sugihara, Fractals, A user"s guide for the national Sciences, Oxford University Press, Oxford, England (1993).

[6] Jost B. Jonas, Xuan N. Nguyen, and Gottfried O. H. Naumann, Parapapillary Retinal Vessel Diameter in Normal and Glaucoma Eyes, Investigative Ophthalmology \& Visual Science, Vol. 30, No. 7, 15991603 (1989)

[7] Mandelbrot.B, The Fractal Geometry of Nature, San Francisco, W.H.Freeman (1983).

[8] Nonnenmacher.T.F, Fractals in Biology and Medicine (1993).

[9] Rybaczuk.M, Kediza.A, Blaszczyk.E, Fractal Description of Cerebellum Surface During Fetal Period, Folia Morphol, 55, 434-436 (1996).

[10]Rybaczuk.M, Kediza.A, Andrzejak.R, Fractal Dimension Of Human Brain Cortex Vessel During The Fetal Period, Med Sci Monit, 8(3) (2002).

[11]Rybaczuk.M, Kediza.A, Paradowski.L, Fractal Characteristic of brain vessel microangioarchitecture during the fetal period, Med Sci Monit, 8(8), MT 145152 (2002).

[12] Suwa.N, Takahashi.T, Morphological and morphometrical analysis of circulation in hypertension and ischemic kidney, Urban and Schjwarzenberg (1971).

[13] Wilkinson.D and Willemsen.J.F, Invasion Percolation, A new form of Percolation Theory. J.Phys.A, 33653376 (1983). 